\title{
RNA interference targeting UL25 gene as a gene therapy approach against BHV-1 virus
}

\section{Azam Mokhtari ( $\nabla$ a.mokhtari@alumni.ut.ac.ir)}

Shahrekord University https://orcid.org/0000-0003-0139-1456

\section{Elaheh Amjadimanesh}

Shahrekord University

\section{Behnaz Saffar}

Shahrekord University

\section{Sahand Shams}

Shahrekord University

\section{Research article}

Keywords: IBRV, RNAi, shRNA, BHV-1, UL25, lentivector

Posted Date: June 19th, 2020

DOl: https://doi.org/10.21203/rs.3.rs-31534/v1

License: (9) This work is licensed under a Creative Commons Attribution 4.0 International License. Read Full License 


\section{Abstract}

Background: In recent decades RNAi has become a novel and effective tool to silence gene expression. The expression of shRNAs against target genes has now become a standard and powerful strategy for antiviral therapeutic approaches. Infectious bovine rhinotracheitis (IBR) is a domestic and wild ruminant viral disease that caused by bovine herpesvirus-1. Occasionally, its entry into the herd may cause vigorous economic damage due to losing weight, reduced output and limitation on livestock international trade. Vaccines against the disease are not fully effective and the need for an effective therapeutic approach is highly felt.

In the present study an RNAi- based gene therapy was designed and implemented. Due to the essential role of UL25 gene for $B H V-1$ replication.

Method: the sequence of UL25 gene was purposed for designing shRNA molecules. The UL25 gene sequence was extracted from the NCBI database and suitable shRNA molecules were purposed by using online soft wares. Three recombinant lentivira/ vectors expressing short hairpin RNAs (shRNAs) versus the UL25 gene of IBRV were constructed and co-transfected into HEK 293T cells. The effectiveness of designed shRNA molecules was assayed by calculating TCID50 titers and observation of BHV-1 cytopathic effects.

Results: The final calculations showed that all shRNAs had antiviral effects. In the TCID50 test, the shRNAs 1, 2 and 3 sufficiently decreased BHV1 output in comparison with the control groups (almost $98.22 \%, 99.63 \%$ and $99.54 \%$, respectively than cells with IBRV inoculation and nearly $90.84 \%, 97.9 \%$ and $96.84 \%$, respectively than cells with ORFV and scrambled vector inoculation). ShRNA-2 achieved maximal inhibition of viral replication.

Conclusion: The results indicated that shRNAs targeting the UL25 gene showed considerable antiviral attributes and reduced IBRV multiplication in MDBK cells. In conclusion; RNA interference can be used as a gene therapy instrument against $B H V$ - 1 virus.

\section{Introduction}

Bovine herpesviruses- 1 causes a viral disease of domestic and wild cows which is called bovine infectious rhinotracheitis (Turin et al., 1999). The major host of the virus is cattle, but other small ruminants may also be infected. After high fever, anorexia, sudden depression, the signs of respiratory infections, abortion in pregnant animals, conjunctival rhinorocyte and traumatic inflammation may occur. Although $B H V$-1 infection isn't a common life-threatening pathogen, it is a risk factor for the expansion of additive bacterial infections and ultimately causes severe economic losses due to the reduction of healthy products and the limitation of international trade (Biswas et al., 2013).

Bovine herpesvirus- 1 belongs to the genus Varicellovirus, subfamily Alphaherpesvirinae and the family Herpesviridae. The virus genome is a double-stranded DNA with the size of $135301 \mathrm{bp}$ that is divided into 
4 segment such as a unique long (UL) segment, a unique short (US) segment and two inverted repeat region that named inverted internal repeat (IR) and terminal repeat (TR) sequences. Two isomeric forms of the viral genome organized by UL, US and inverted repeat regions. 73 open reading frames (ORF) that encode for 70 proteins have been identified in BHV-1 genome (Bowman et al., 2006). The UL 25 gene is one of the 40 nuclear genes of the virus and is conserved in the all members of herpesvirinae subfamily (Bowman et al., 2006). This gene is located at the position 60602-62398 of the BHV-1 genome and codes a $63 \mathrm{KDa}$ tegument protein with 598 amino acids which is necessary in the accumulation and packaging of genomic DNA into the capsid structure (Desloges et al., 2001).

$B H V-1$ virus has distributed into three subtypes such as $B H V-1.1, B H V-1.2$, and $B H V-1.3 . B H V-1.1$ often is linked to the respiratory manifestation of the disease (IBR), $B H V-1.2$ causes reproductive infections and $B H V-1.3 / B H V-5$ is a neuropathogenic agent that is associated with neurological disorders (Muylkens et al., 2007). It is worth noting that all subtypes are antigenically equivalent and indistinguishable with typical serological tests (Keuser et al., 2004).

Over the years, continuous researches have been conducted to discover effective anti- IBR vaccines or therapeutic agents. Although, currently, several vaccines are available for the disease; but none of them are completely specific and effective. Therefore, the necessity of providing efficient and specific anti $B H V$ 1 preventive/ therapeutic methods is felt (Song et al., 2016).

The RNAi pathway is a process in which two strands RNA molecules with specific length and secondary structure degrade the homologous RNA targets or suppress the expression of their complementary genes. This pathway in the cell is naturally aimed to protect the genome against external genetic threats such as viral genes, transgenes and internal threats like transposons. Furthermore, it is responsible for regulate gene expression and development (Ma et al., 2007).

Nowadays the RNA interference pathway has become a mere biological phenomenon to a powerful therapeutic tool for treating a wide range of diseases, including viral infections. Ease of use, rapidity, excellent performance and remarkable specificity when exerted at various phases of virus-host interplay are some of the potential advantages of using the RNAi pathway as an antiviral approach over traditional methods such as antiviral drugs or vaccines (Fischer et al., 2004).

RNAi is supplied to the cell from short interfering RNA (siRNA) or short hairpin RNA (shRNA) to down regulate the expression of purposed genes. Many studies have shown shRNA offers advantages in silencing the target genes such as stability, cost-effectiveness and ease of delivery. The shRNA expression cassettes are consistently integrated into the host cell genome and suppress expression of the target gene by homologous mRNA degradation without change in other mRNAs. Thus, RNAi seems to be an appropriate option as a therapeutic method for protecting plant and animal species against various viruses (Rao et al., 2009; Narute et al., 2009).

In the present study, due to the essential task of the UL25 gene in BHV-1 multiplication [11], this gene sequence was chosen to design shRNA molecules against IBR disease. 


\section{Material And Methods}

\subsection{Cell lines and viruses:}

MDBK cells (ATCC Number: CCL-22) and Human embryonic kidney 293T cells (ATCC Number: CRL-1573) cultured in Dulbecco's modified Eagle's medium (DMEM; Gibco CO) supplemented with 10\% Fetal bovine serum (FBS; Gibco CO) and 1\% penicillin/streptomycin (Pen-Strep, Sigma CO). IBRV was kindly provided by Dr. Darounkola (Institute of Virology, Faculty of Veterinary Medicine, University of Zürich) and was propagated in MDBK cells

\subsection{Design of anti- BHV1-UL25 shRNA molecules}

For a better efficiency, shRNA molecules must be designed to disrupt expression of conserved regions of the viral target gene. So by using NCBI database, the sequence of $B H V-1-U L 25$ gene (Accession number: AJ004801.1) was obtained and was aligned with the sequence of this gene in the other strains of virus by CLUSTAL OMEGA online software. Therefore the most homologous regions were selected for further analyzes. Several shRNA molecules were designed using three online web servers such as WI siRNA selection program, BLOCK-IT RNAi designer and www.invivogen.com/siRNA-wizard. The sequences of proposed shRNA molecules were matched to the target gene sequence to find those which target the conserved regions. Since it is important that shRNA molecules should easily access to the UL25 mRNA, CLC Genomic Work brench software was applied to predict the secondary structure RNA of UL25 gene and the position of shRNA molecules that are related to the conserved regions in this structure. Selected sequences were submitted to a BLAST search against the cattle genome sequence to ensure that the host genome was not targeted. After the subsequent investigation of optimal CG percentage and off-target effects, three shRNA sequences were finalized. The sequences of the cut-off site of the BamH and EcoRI restriction enzymes were placed on both sides of shRNA sequences and TCAAGAG were placed as a loop sequence. Afterwards, the oligonucleotides were synthesized by Bioneer Co.

\subsection{Preparation of anti- BHV1-UL25 shRNAs}

The proposed shRNA molecules were synthesized as single-strand oligoes by Bioneer Co. and were annealed with each other. In order to annealing, a mixture of $1 \mu \mathrm{L}$ of sense oligo $(200 \mu \mathrm{M}), 1 \mu \mathrm{L}$ of antisense oligo $(200 \mu \mathrm{M})$, and $2 \mu$ l of sterile deionized water were mixed, then the mixture was placed in a boiling water at $95^{\circ} \mathrm{C}$ for 4 minutes to remove all possible secondary structures. Finally, it was placed at room temperature for 10 minutes to form the desired double strands.

\subsection{Production of lentivectors expressing anti- BHV1-UL25 shRNAs}

2.4.1. Lentiviral Plasmaids. In the present study, psPAX2 (10703 bp); a packaging construct that containing gag and po/genes, pMD2G; an env (VSV-G) plasmid (5824 bp) and a self-inactivating (SIN) transfer vector plasmid pCDH-CMV-MCS-EF1-CGFP-T2A-Puro (CD513B-1,8220 bp) were used to generate third-generation lentivira/ vectors. pEZX-MR03 containing eGFP gene (7945 bp) was applied as control plasmid (all the plasmid were kindly provided from Bonbiotech company(Iran)). 
2.4.2. Production of Lentiviral transfer Plasmaid: To construct lentiviral plasmids carrying shRNA sequences, pCDH-CMV-MCS-EF1-CGFP-T2A-Puro lentiviral plasmid was double- digested with BamHI (Roche, Germany,) and EcoRI (Roche, Germany). The Ligation reaction was carried out with T4 10X ligation buffer (Fermentas, Germany, Cat. No.: B69), T4 DNA ligase (10U) (Fermentas, Germany), synthetized shRNA oligos and lentiviral plasmid and were transformed in the E.coli DH5a. Then, colony PCR reaction was performed using the general primers of PCDH-CMV-MCS-EF1-cGFP-T2A-Puro lentiviral plasmid. The sequences of primers were as follows: CMV-F: AATGGGCGGTAGGCGTGTA -3'and EF1-R: 5'GGACTGTGGGCGATGTG -3 '. The PCR thermal cycle program was consisted of denaturation at $95^{\circ} \mathrm{C}$ for $5 \mathrm{~min}$ followed by 30 cycles at $95^{\circ} \mathrm{C}$ for $30 \mathrm{~s}, 55^{\circ} \mathrm{C}$ for $40 \mathrm{~s}$ and $72^{\circ} \mathrm{C}$ for $60 \mathrm{~s}$, followed by a final extension at $72{ }^{\circ} \mathrm{C}$ for 10 minutes. Finally the recombinant lentivira/ plasmids were sequenced by Bioneer Co.

2.4.3. Co- transfection of lentiviral plasmids: After two passages of HEK $293 \mathrm{~T}$ cells the cells were cotransfected with a mixture of $21 \mu \mathrm{g}$ psPAX,10.5 $\mathrm{g}$ pMD2.G and $21 \mu \mathrm{g} \mathrm{pCDH}$ (or pEZX-MR03 as a mock) using Ca-Po4 reagent according to the bonbiotech company's guidelines. The co-transfection was performed in 10-cm plates similar to the conditions mentioned in manufacturer's instructions in order to gain a confluency of approximately 70-80\% (Zufferey et al., 2000). 48 and $72 \mathrm{~h}$ post transfection, lentivectors in the supernatant were harvested by centrifugation $(1000 \mathrm{~g}, 15 \mathrm{~min})$ and were kept in $-70{ }^{\circ} \mathrm{C}$ until the infection of MDBK cells. The transfected HEK-293T cells were photographed under a fluorescent microscope for monitoring EGFP expression.

\subsection{Lentivector transduction, challenge with $B H V-1$ :}

MDBK monolayers ( $5 * 10^{3}$ cells in 96 -well plate, $70 \%$ confluency) were propagated and inoculated with $280 \mu \mathrm{L}$ of lentiviruses expressing shRNAs ( $\mathrm{MOI}=0.5$ ), diluted in $1 \mathrm{~mL}$ of DMEM medium supplemented by $3 \%$ FBS. After 12 hours, the medium of each well was replaced with fresh DMEM supplemented with $3 \%$ FBS. At $48 \mathrm{~h}$ and $72 \mathrm{~h}$ after infection, MDBK cells were observed using a fluorescent microscope and EGFP expression was monitored for confirmation of shRNA- expression.

Subsequently, transduced MDBK cells were infected by $B H V-1$ and monitored for the development of cytopathic effects. Finally TCID 50 titers were calculated for the wells that infected with the $B H V-1$ and shRNA expressing lentivectors and positive (cells infected with only $B H V-1$ or $B H V-1$ and scrambled lentivector) and negative controls (cells infected with scrambled lentivectoror without viral infection).

\section{Results}

\section{Design of shRNAs:}

Several shRNA molecules were designed using online soft wares and three of them were selected based on defined parameters and secondary structure status. The sequences of three anti- BHV1-UL25 shRNA oligonucleotides have been listed in the table 1. Secondary structure of viral mRNA for UL25 gene was predicted by CLC software and the interaction sites with the designed shRNAs are shown in figure 1. 
<Figure 1 near here>

\section{ShRNA expressing lentivector production:}

After co-transfection of transfer and mock lentivira/plasmids with psPAX2 and pMD2G, the expression of the EGFP reporter protein was evaluated by fluorescent microscopy and since the shRNA sequence was at the upstream of EGFP gene, the production of lentivectors that expressed shRNAs was confirmed (Fig. 2).

$<$ Figure 2 near here>

\section{Challenge of $B H V-1$ and lentivector expressing shRNAs:}

After lentivira/ vectors infection, $48 \mathrm{~h}$ and $72 \mathrm{~h} \mathrm{pi,} \mathrm{MDBK}$ cells were observed using a fluorescent microscope and EGFP expression was monitored for confirmation of shRNA- expression (Fig. 3).

The development of cytopathic effects in MDBK cells was monitored after inoculation of $B H V-1$. The cytopathic effects observed in the cells were co- infected with BHV-1 and shRNA expressing lentivectors and compared with positive controls (the cells were infected with only $B H V-1$ and those were infected by $B H V-1$ and scrambled lentivector) and normal cells as negative control. The results showed that cells infected with anti-BHV-1 shRNAs visibly reduced CPE $72 \mathrm{~h}$ after the infection, compared to cells of positive control group (Fig. 4).

$<$ Figure 3 near here $>$

$<$ Figure 4 near here>

\section{TCID50 assay:}

The TCID50 assay was performed to evaluate the efficiency of shRNAs to inhibit IBRV replication. The final calculations showed that in the TCID50 test, the shRNAs 1,2 and 3 also significantly reduced virus yield compared to the control groups (approximately $98.22 \%, 99.63 \%$ and $99.54 \%$, respectively compared to cells infected with ORFV and approximately $90.84 \%, 97.9 \%$ and $96.84 \%$, respectively compared to cells infected with ORFV and scrambled vector) (Figure 5).

\section{Discussion}

In spite of many attempts have been made to discover and apply effective vaccines or antiviral agents, the disease remain prevalent and the effectiveness of common available vaccines isn't ideal (Romera it al., 2014; Song et al., 2016)..

It is established that treatment based on altered target gene expression and employing a natural intracellular pathway has fewer side effects than other therapies. Therefore, RNAi has been considered as a powerful strategy for the treatment of many viral diseases in recent years (Perrimon et al., 2010). 
Despite the advantages of using siRNA molecules for therapeutic applications against acute viral infections, the use of shRNA molecules that triggers a more stable gene expression and less off- target effects seems to be more appropriate. On the other hand, so far, some features of lentivira/ vectors include extensive tissue tropism, persistent gene expression and low carcinogenic risk has been reported in the in vivo conditions (Kafri et al., 2000). Considering the advantages of lentivectors and shRNA molecules, in the present study lentivector- based shRNAs were selected to suppress gene expression.

UL25 gene is one of the 40 core IBRV genes that is conserved among all members of the alpha-, beta-, and gamma herpesvirinae subfamilies. Its role in DNA packaging and attainment of the tegument layer by the virion is very important and therefore in the present study it UL25 gene was chosen.

In recent years many studies have been conducted for applying RNAi pathway to suppress gene expression in order to determine the role of unknown genes or providing antiviral strategies against many viruses including herpesviridae. For example, Qingmei Jia and Ren Sun (2003) designed siRNA molecule against $O R F-45$ in the members of gamma herpesvirinae to block virus replication, and demonstrated the essential role of the ORF-45 to efficient viral production (Jia et al., 2003).

Lu" der Wiebusch et al. (2004) used siRNA-UL54 to down regulate the expression of UL54 gene in human cytomegalovirus (HCMV) and observed a 12-fold decrease in virus titers after $96 \mathrm{~h}$ of infection (Wiebusch et al., 2004).

Ren Zhe et al. (2008) designed siRNAs targeting the UL39 gene of HSV-1 and showed that synthetic siRNA were able to effectively prevent target gene expression and virus replication. Therefore, they suggested the use of RNAi pathway as a powerful genetic strategy to inhibition of $\mathrm{HSV}-1$ replication (Zhe et al., 2008).

Wilkes et al. (2009) designed siRNAs against the glycoprotein D gene in feline herpesvirus 1 (FHV-1) and showed decrease of $84 \%$ and $77 \%$ in target viral titer (Wilkes et al., 2009).

Narute et al. (2009) synthesized two siRNA molecules against BHV-1- UL25 and inhibited its replication in MDBK cells. The reduction of viral titers ( $11 \%$ and $40 \%)$ determined in the cell culture system by TCID50 titration method (Narute et al., 2009).

Unfortunately we didn't find any reports of suppressing the gene expression of IBRV using lentiviral mediated RNAi. However, there are a number of study in which lentiviruses have been applied to induce RNAi against other members of herpesviridae. For example wang et al. (2017) with lentivirus-mediated RNAi suppressed $L M P 2 A$ and described E. Barr-associated GT38 cell line was inhibited in vitro (Wang et al., 2017). Song et al. (2016) designed two shRNA molecules delivered by lentivectors against $g D$ to IBRV replication in MDBK cells. After performing TCID50 assay, the results indicated that silencing viral $g D$ gene expression was successful (Song et al., 2016). In the present study three shRNAs against IBRVUL25 gene were constructed and expressed via lentivira/ vectors. We demonstrated the designed shRNAs could successfully inhibit IBRV multiplication in vitro. As demonstrated by TCID50 assay, shRNA2, 
shRNA3 and shRNA1 downregulated the IBRV replication in the amount of 97.9, 96.84 and 90 percent, respectively.

Finally, it should be stated although the results of the present study verified the feasibility of a suitable treatment against IBRV infection via RNAi pass way in the cell culture system, the use of this method in the in vivo system requires further investigation and studies.

\section{Declarations}

Declarations of interest: none

Submission declaration and verification: This work has not been published previously and it is not under consideration for publication elsewhere. Its publication is approved by all authors and tacitly or explicitly by the responsible authorities where the work was carried out. If the manuscript accepted, it will not be published elsewhere in the same form, in English or in any other language, including electronically without the written consent of the copyright-holder.

Use of inclusive language: Authors ensure that writing is free from bias

Authors' contributions: EA and SS designed shRNAs and performed the cloning and cell culture tests. AM and BS calculated and interpreted the results. AM performed the final interpretation and wrote the manuscript.

Role of the funding source: This work was supported by grants from Shahrekord University (97GRN1M1801).

\section{Acknowledgments:}

This work was supported by Shahrekord University (grant number: 96GRD1M1801).

\section{Conclusion}

The results of the present study showed that anti BHV1- UL25 shRNAs had significant antiviral features and suppressed IBRV multiplication in MDBK cells, so lentivirus-mediated RNAi can be used as an effective approach to inhibit $B H V$-1 infection.

\section{References}

1. Biswas S, Bandyopadhyay S, Dimri U, H. Patra P. Bovine herpesvirus-1 (BHV-1)-a re-emerging concern in livestock: a revisit to its biology, epidemiology, diagnosis, and prophylaxis. Veterinary Quarterly. 2013 Jun 1;33(2):68-81

2. Bowman BR, Welschhans RL, Jayaram H, Stow ND, Preston VG, Quiocho FA. Structural characterization of the UL25 DNA-packaging protein from herpes simplex virus type 1. Journal of 
virology. 2006 Mar 1;80(5):2309-17.

3. Desloges N, Boucher H, Simard C. Transcriptional and translational expression kinetics of the UL25 homologue of bovine herpesvirus 1.1. Archives of virology. 2001 Sep 1;146(9):1693-704.

4. Fischer LT, James QY. RNAi, a new therapeutic strategy against viral infection. Cell research. 2004 Dec;14(6):460-6.

5. Jia Q, Sun R. Inhibition of gammaherpesvirus replication by RNA interference. Journal of virology. 2003 Mar 1;77(5):3301-6.

6. Kafri T, Van Praag H, Gage FH, Verma IM. Lentiviral vectors: regulated gene expression. Molecular Therapy. 2000 Jun 1;1(6):516-21.

7. Keuser V, Schynts F, Detry B, Collard A, Robert B, Vanderplasschen A, Pastoret PP, Thiry E. Improved antigenic methods for differential diagnosis of bovine, caprine, and cervine alphaherpesviruses related to bovine herpesvirus 1. Journal of clinical microbiology. 2004 Mar 1;42(3):1228-35.

8. Ma Y, Chan CY, He ML. RNA interference and antiviral therapy. World Journal of Gastroenterology: WJG. 2007 Oct 21;13(39):5169

9. Muylkens B, Thiry J, Kirten P, Schynts F, Thiry E. Bovine herpesvirus 1 infection and infectious bovine rhinotracheitis. Veterinary research. 2007 Mar 1;38(2):181-209.

10. Narute PS, Raut AA, Saini M, Rai A, Gupta PK. Inhibition of Bovine herpesvirus 1 multiplication in MDBK cells by small interfering RNAs. Acta virologica. 2009 Jan 1;53(3):203.

11. Perrimon N, Ni JQ, Perkins L. In vivo RNAi: today and tomorrow. Cold Spring Harbor perspectives in biology. 2010 Aug 1;2(8):a003640.

12. Rao DD, Vorhies JS, Senzer N, Nemunaitis J. siRNA vs. shRNA: similarities and differences. Advanced drug delivery reviews. 2009 Jul 25;61(9):746-59.

13. Romera SA, Puntel M, Quattrocchi V, Zajac PD, Zamorano P, Viera JB, Carrillo C, Chowdhury S, Borca MV, Sadir AM. Protection induced by a glycoprotein E-deleted bovine herpesvirus type 1 marker strain used either as an inactivated or live attenuated vaccine in cattle. BMC veterinary research. 2014 Dec 1;10(1):8.

14. Song $L$, Zhang $H$, Wang $H$, Hou P, Xia X, He H. RNA interference targeting glycoprotein D inhibits infectious bovine rhinotracheitis virus replication in MDBK cells. The Thai Journal of Veterinary Medicine. 2016;46(3):373-80.

15. Turin L, Russo S, Poli G. BHV-1: new molecular approaches to control a common and widespread infection. Molecular Medicine. 1999 May;5(5):261-84.

16. Wang F, Chen W, Liu P, Zhou J, Liu B, Ye W, Wang W, Shen X. Lentivirus-mediated RNAi knockdown of LMP2A inhibits the growth of the Epstein-Barr-associated gastric carcinoma cell line GT38 in vitro. Experimental and therapeutic medicine. 2017 Jan 1;13(1):187-93.

17. Wiebusch L, Truss M, Hagemeier C. Inhibition of human cytomegalovirus replication by small interfering RNAs. Journal of General Virology. 2004 Jan 1;85(1):179-84. 
18. Wilkes RP, Kania SA. Use of interfering RNAs targeted against feline herpesvirus 1 glycoprotein D for inhibition of feline herpesvirus 1 infection of feline kidney cells. American journal of veterinary research. 2009 Aug;70(8):1018-25.

19. Zhe R, Mei-Ying Z, Kitazato K, Kobayash N, Qin-Chang Z, Pei-Zhuo Z, Zhi-Rong Y, Yi-Fei W. Effect of siRNA on HSV-1 plaque formation and relative expression levels of UL39 mRNA. Archives of virology. 2008 Jul 1;153(7):1401-6.

20. Zufferey R, Trono D. Production of high-titer lentiviral vectors. Current protocols in human genetics. 2000 Aug;26(1):12-0

\section{Tables}

Table 1: The sequences of designed anti- UL25 shRNA oligonucleotides

\begin{tabular}{|ll|}
\hline Name & ShRNA sequence \\
\hline ShRNA-1 & AATTCGCCATGAAGGTTCGAGACATGTCAAGAGCATGTCTCGAACCTTCATGGCG \\
\hline ShRNA-2 & AATTCAATTTCGCTCGTCCGGTACAATCAAGAGTTGTACCGGACGAGCGAAATTG \\
\hline ShRNA-3 & AATTCGCCCAGGCAAGCAAACTCTAATCAAGAGTTAGAGTTTGCTTGCCTGGGCG \\
\hline
\end{tabular}

\section{Figures}



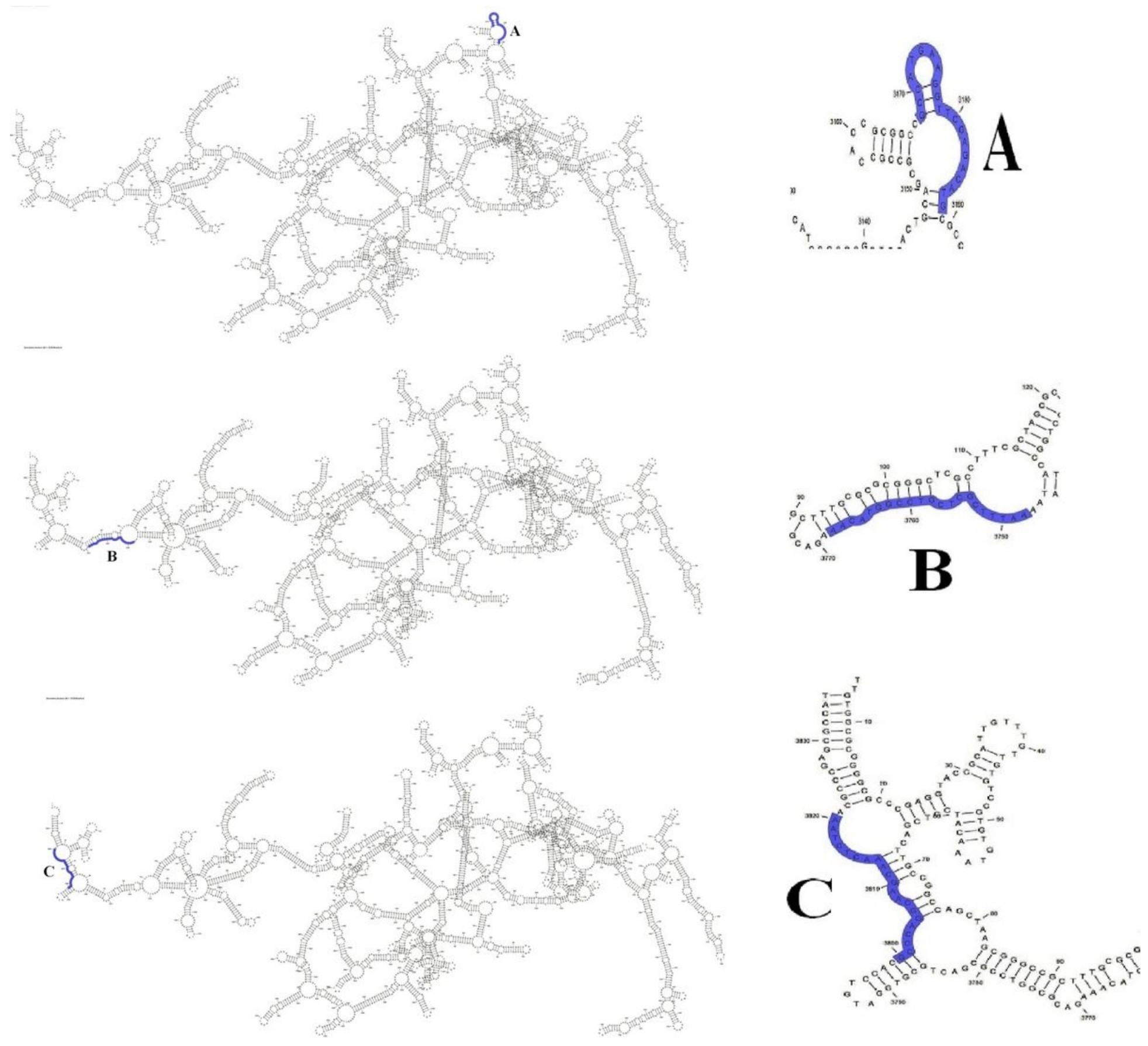

Figure 1

Matching of designed shRNAs with BHV-1 mRNA Secondary structure of UL25 shRNA Target Sites (black) on the BHV1-UL25 mRNA Secondary Structure Predicted Using CLC (A: BHV-ShRNA1, B: BHVShRNA2, C: BHV-ShRNA3) 


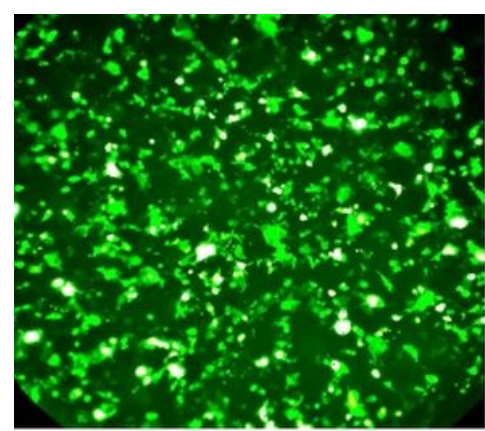

A

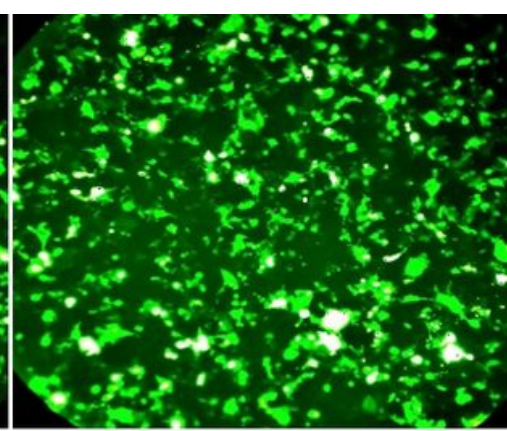

$\mathrm{B}$

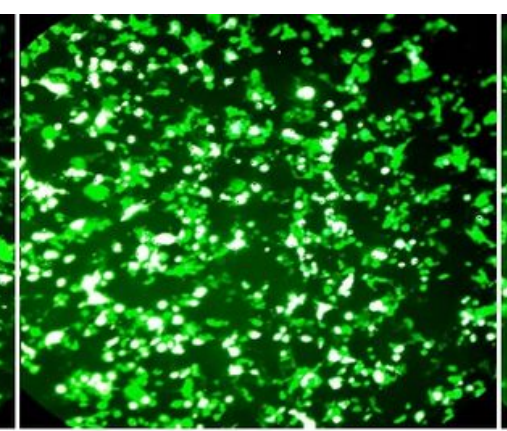

C

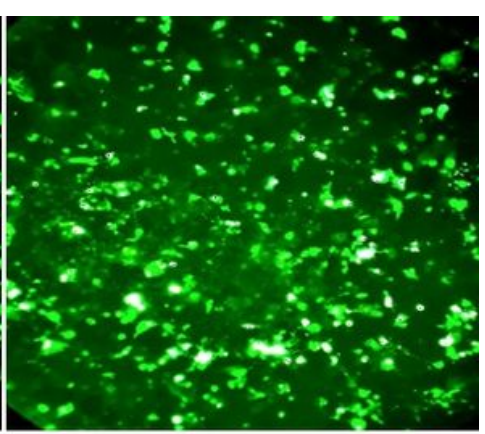

D

\section{Figure 2}

EGFP expression after Co-transfection of HEK293T cells EGFP expression by transfected cells with psPAX and pMD2.G as packaging vectors and $\mathrm{pCDH}$ carrying BHV-ShRNA1, $\mathrm{pCDH}$ carrying BHV-ShRNA2, pCDH carrying BHV-ShRNA3 and pEZX-MR03 (as a mock), respectively.

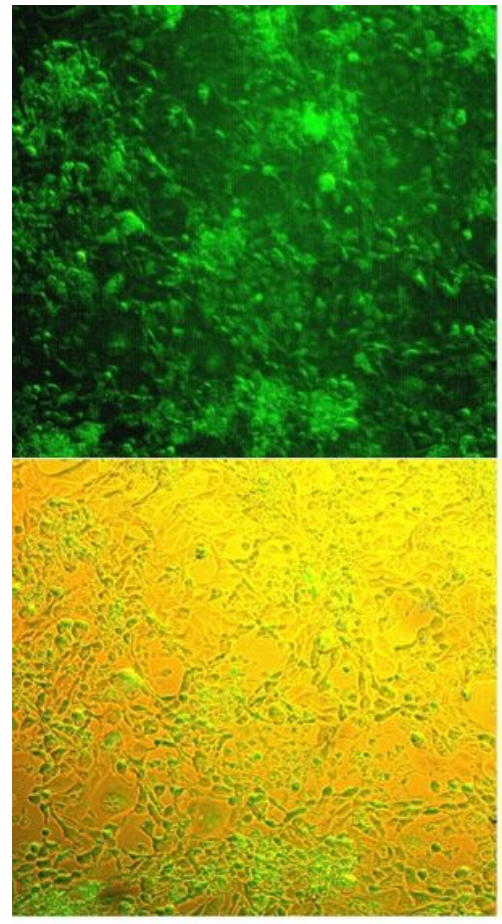

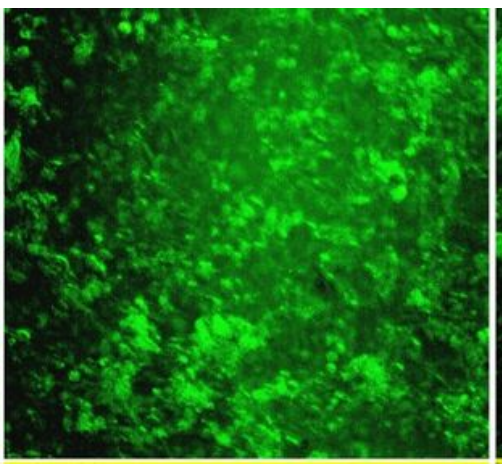
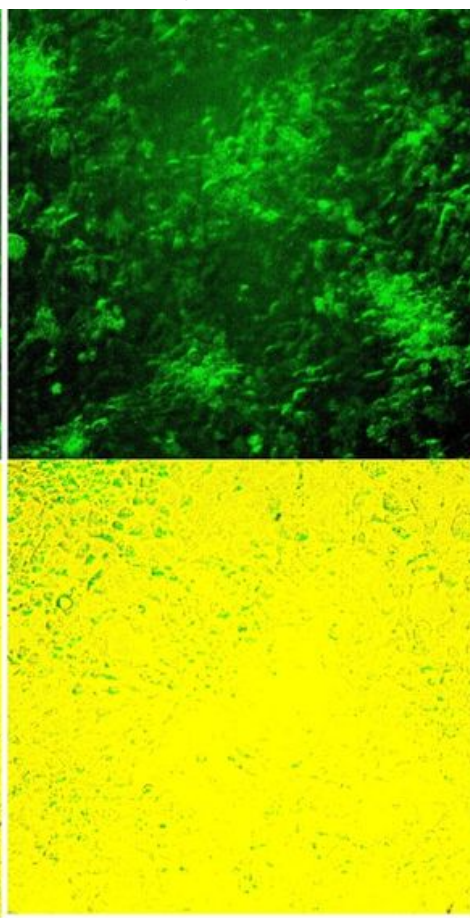

c

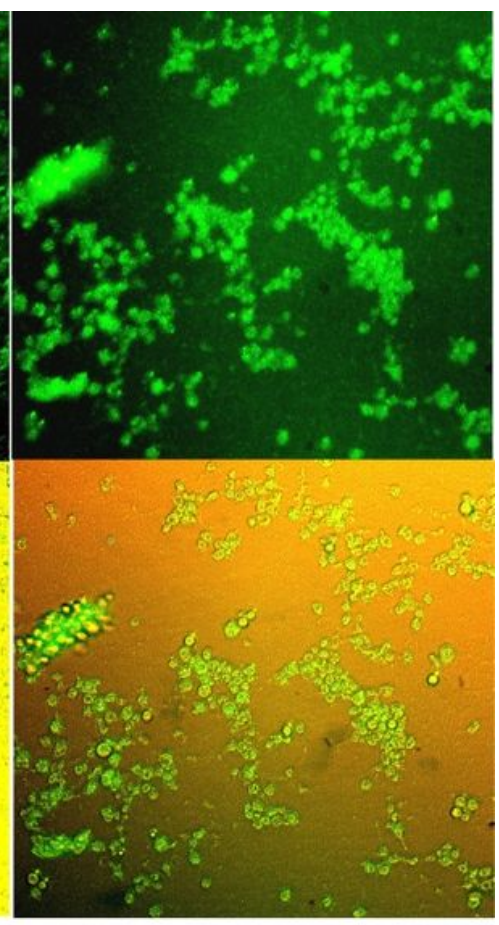

D

Figure 3

GFP expression after infection MDBK cells with lentivectors GFP expression by infected MDBK cells with lentivector expressing BHV-ShRNA3, BHV-ShRNA2, BHV-ShRNA1 and scrambled lentivector respectively and the same pictures with the light microscope. 


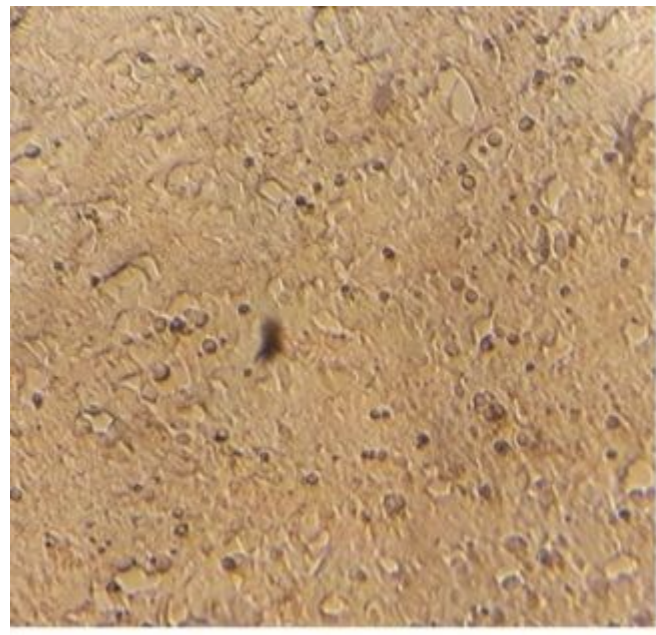

A

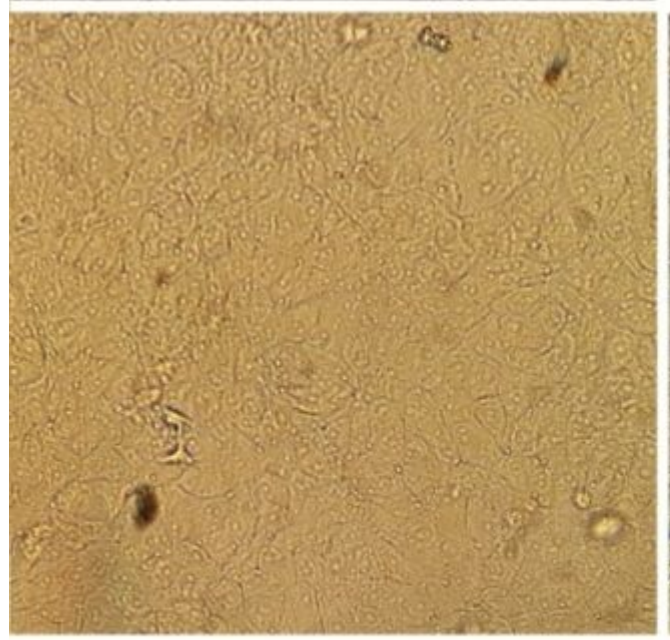

D

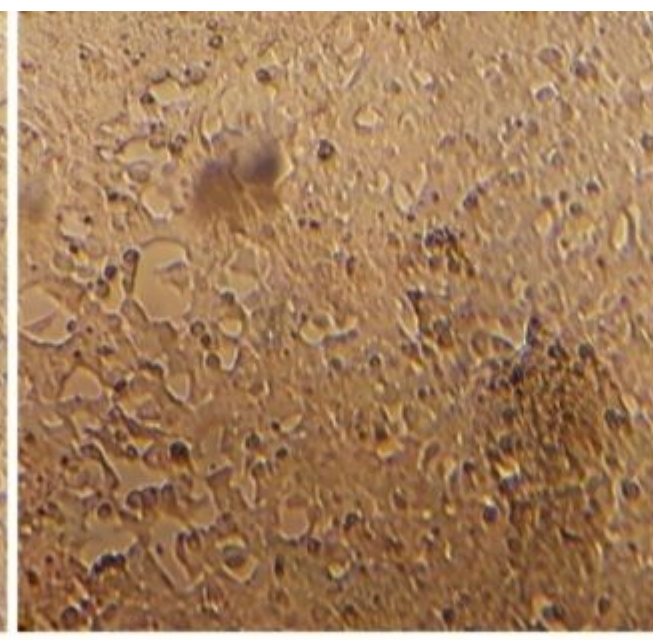

B

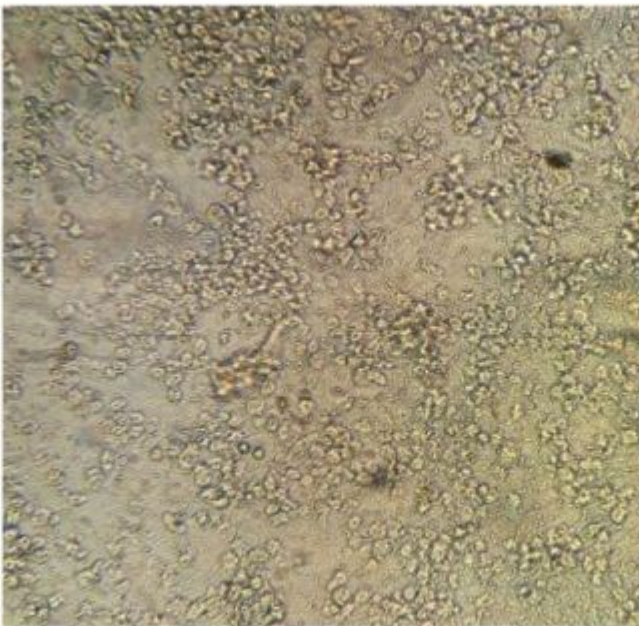

$E$

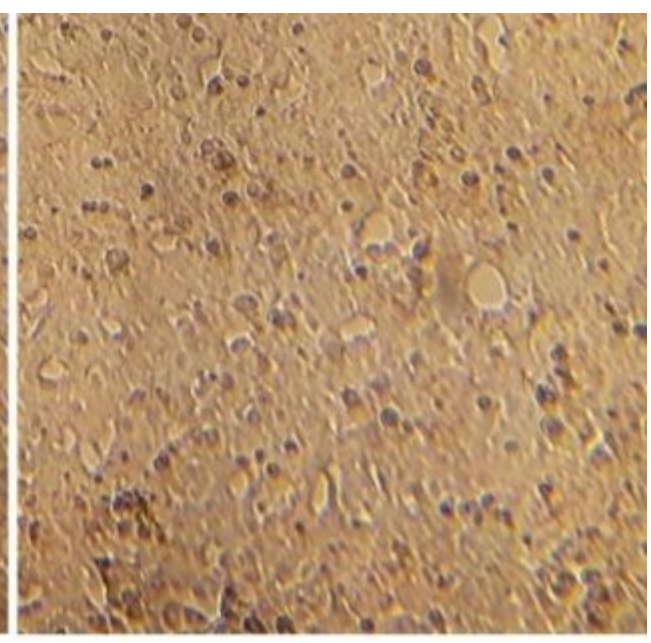

C

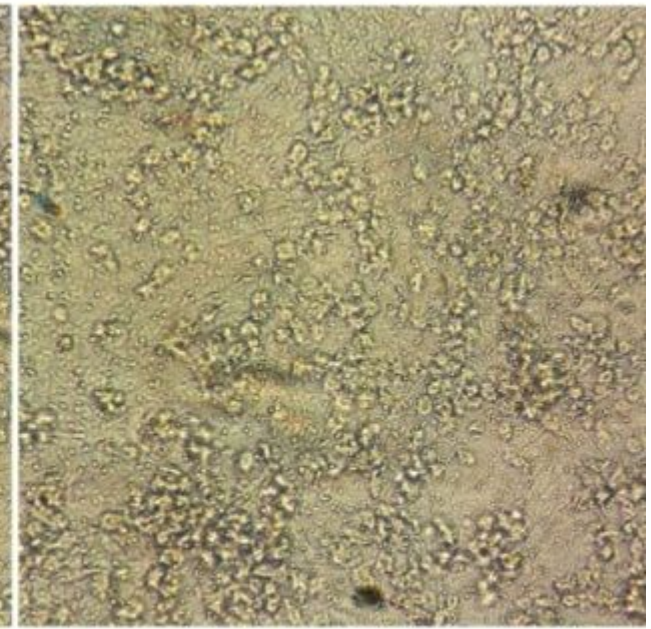

$\mathrm{F}$

\section{Figure 4}

Challenge of BHV-1 and ShRNAs in MDBK cells A, B and C: Cells co-infected with BHV-1 and shRNA-2, shRNA-1, shRNA-3 recombinant lentiviral vectors, respectively, D: Cells without virus inoculation, E: Cells infected with BHV-1, F: Cells co- infected with BHV-1 and scrambled lentivector.
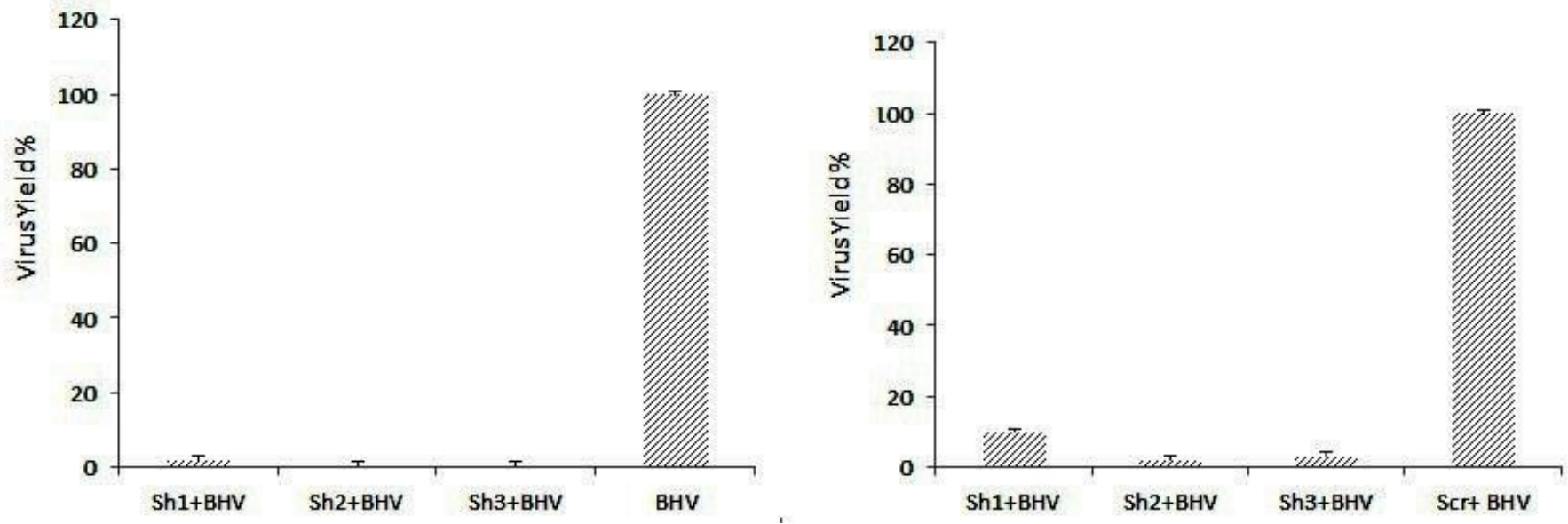


\section{Figure 5}

TCID50 assay The titration of virus was detected at $48 \mathrm{~h}$ pi. The viral yields were measured by the ratio of TCID50 of expressed shRNAs to that of controls (cells infected with BHV-1 and those infected by scrambled vector). Error bars show standard deviation of three independent experiments. The shRNAs 1 , 2 and 3 reduced virus yield compared to cells infected with BHV-1 (Left chart) and compared to cells infected with BHV-1 and scrambled vector (Right chart). 